\title{
Growth Hormone Stimulation Tests in the Differential Diagnosis of Parkinson's Disease
}

\author{
Maria Teresa Pellecchia, MD; Rosario Pivonello, MD; Annamaria Colao, MD and Paolo Barone, MD
}

\begin{abstract}
Idiopathic Parkinson's disease (IPD) is a common neurodegenerative disorder whose differential diagnosis from other forms of atypical parkinsonism, for instance multiple system atrophy (MSA) or progressive supranuclear palsy, may be difficult, especially in the early stages. Growth hormone stimulation tests have been recently reported to be useful in the differential diagnosis between IPD and MSA. Both clonidine, an $\alpha_{2}$-adrenoceptor agonist, and arginine, an amino acid activating the cholinergic system, have been used to assess growth hormone response in patients with IPD and MSA. This review summarizes the results of several studies and discusses the validity of these tests in the differential diagnosis of parkinsonisms.
\end{abstract}

Keywords: Arginine; Clonidine; Growth hormone; Multiple system atrophy; Parkinson's disease

$I_{\text {d }}$

diopathic Parkinson's disease (IPD) is a slowly progressive neurodegenerative disorder whose cardinal clinical signs are bradykinesia, rest tremor, rigidity and gait/balance impairment. Community-based studies showed that it is one of the most common neurodegenerative disorders of the elderly and accounts for about $80 \%$ of all parkinsonisms. ${ }^{1-3}$ Pathologically, IPD is characterized by loss of pigmented neurons and gliosis, most prominently in the substantia nigra pars compacta and locus coeruleus, as well as by the presence of typical eosinophilic cytoplasmic inclusions (the Lewy body) in degenerating neurons. ${ }^{4}$

Since diagnosis of IPD is mostly dependent on clinical criteria, some cases may be misclassified. In one series, $20 \%$ of patients diagnosed in life as having IPD had some other diagnosis, usually one of the atypical parkinsonian syndromes, at autopsy. ${ }^{5}$

Multiple system atrophy (MSA) is a sporadic neurodegenerative disorder clinically characterized by various combinations of parkinsonian, cerebellar, autonomic and pyramidal signs. ${ }^{6}$ Postmortem examination of MSA patients reveals striatonigral and/or olivopontocerebellar degeneration and typical glial cytoplasmic inclusions. ${ }^{7}$ Two major motor presentations can be distinguished clinically: MSA-P occurs in $80 \%$ of cases and is mainly characterized by parkinsonian features, and MSA-C is less frequent ( $20 \%$ of cases) and mainly characterized by cerebellar features. ${ }^{6}$
It is estimated that $8 \%$ of all patients presenting with parkinsonism will eventually develop MSA. ${ }^{8}$ Differential diagnosis of MSA and IPD may be difficult due to several overlapping features, such as rest tremor or asymmetrical akinesia and rigidity. ${ }^{8}$ Most patients with MSA have generalized autonomic dysfunction, but this may appear only in late stages of the disease, so that an accurate early diagnosis is difficult. Indeed, about $25 \%$ of patients with pathological evidence of MSA had a clinical diagnosis of IPD in life. ${ }^{9}$ The differential diagnosis between MSA and IPD is relevant, as MSA is characterized by a poorer response to drug treatment, a wide range of complications that increase morbidity and mortality and a worse prognosis than IPD. The difficulty in differentiating MSA from IPD is reflected by the number of instrumental tests used to support a clinical diagnosis. Autonomic function tests, sphincter electromyography, routine and diffusion-weighted magnetic resonance imaging, functional imaging by use of positron emission tomography and single photon emission computed tomography are used, but may be limited in their accuracy (where known) or practicality.

The secretion of growth hormone is regulated through a complex neuroendocrine control system that relies on the functional interplay of two hypothalamic neurohormones, growth hormone-releasing hormone (GHRH) and somatostatin, exerting stimulatory and inhibitory influences, respectively, on growth hormone secretion. GHRH and somatostatin release are modulated by a number of 
Table 1. Comparison of sensitivity, specificity and positive predictive values of the clonidine growth hormone test in multiple system atrophy (MSA) versus idiopathic Parkinson's disease (IPD) in different studies.

\begin{tabular}{|c|c|c|c|}
\hline & Sensitivity & Specificity & Positive predictive value \\
\hline Tranchant et al ${ }^{15}$ & $100 \%$ & $63 \%$ & $50 \%$ \\
\hline Strijks et al ${ }^{16}$ & $73 \%$ & $57 \%$ & $47 \%$ \\
\hline \multicolumn{4}{|l|}{ Lee et $\mathrm{al}^{17}$} \\
\hline MSA-P vs IPD & $78.3 \%$ & $85.7 \%$ & $85.7 \%$ \\
\hline MSA-C vs IPD & $63.6 \%$ & $85.7 \%$ & $82.4 \%$ \\
\hline Pellecchia et al $^{19}$ & $80 \%$ & $75 \%$ & $75 \%$ \\
\hline
\end{tabular}

neurotransmitters, especially the noradrenergic and cholinergic ones. ${ }^{10}$ In particular, the activation of hypothalamic $\alpha_{2}$-adrenoceptors and muscarinic cholinergic receptors induces growth hormone release through stimulation of GHRH and inhibition of somatostatin release, respectively. ${ }^{10,11}$ Therefore, both clonidine, an $\alpha_{2}$-adrenoceptor agonist, and arginine, an amino acid activating the cholinergic system, are currently used in clinical settings as growth hormone secretagogues. ${ }^{12}$

\section{Clonidine Growth Hormone Test (CGHT) in the Differential Diagnosis of IPD and MSA}

CGHT emerged as a promising tool in the differential diagnosis of IPD and MSA in $1997 .{ }^{13}$ Kimber et al $^{13}$ investigated the effects of clonidine on 14 IPD patients, 31 MSA (15 MSA-P, 16 MSA-C) patients and 27 healthy controls. None of the patients had been taking antiparkinsonian drugs. After an overnight fast, a blood sample was taken from an antecubital vein 15 minutes before infusion of $2 \mu \mathrm{g} / \mathrm{kg}$ clonidine in $20 \mathrm{ml}$ saline. Blood samples were then collected every 15 minutes for 1 hour. After clonidine, growth hormone increased in patients with IPD and in controls, but there was no rise in growth hormone concentration in patients with MSA in either the parkinsonian or cerebellar forms. The difference in mean growth hormone increase between IPD and MSA groups was highly significant. Nevertheless, none of the patients with MSA showed clinical or biochemical evidence of somatotropic dysfunction, as indicated by normal plasma concentrations of IGF-I in all MSA patients and normal growth hormone response to levodopa in 9 of them. Individual results were not described and sensitivity and specificity of CGHT in the differential diagnosis of MSA from IPD were not reported. A specific involvement of hypothalamic $\alpha_{2}$-adrenoceptor sites modulating GHRH release in MSA was suggested to explain these findings. ${ }^{13}$

Clarke et al ${ }^{14}$ studied 9 MSA, 8 early untreated IPD and 9 advanced IPD patients treated with levodopa and/or dopamine agonists. Dosing and methodology of the test were the same as the previous study, but the authors failed to find a significant difference in mean growth hormone response to clonidine between the three groups. Individual growth hormone responses to clonidine were not reported.
Additional studies were performed to clarify these conflicting findings and assessing diagnostic accuracy of CGHT in the differential diagnosis of parkinsonism. ${ }^{15-17}$ Tranchant et al ${ }^{15}$ studied 19 IPD and 7 MSA patients and described both individual and mean growth hormone response to clonidine. Most of the patients received long-term treatment with levodopa and/or dopaminergic agonists. Using the CGHT test, Tranchant and colleagues found that growth hormone levels did not increase after clonidine in all $7 \mathrm{MSA}$, but increased in only 12 of the 19 IPD patients, while it remained stable in the other 7 . In this study, CGHT showed a very high sensitivity (100\%) for the diagnosis of MSA, but a poor specificity (63\%).

Strijks et al ${ }^{16}$ performed CGHT in 21 early IPD and 11 MSA-P patients. None of the patients had taken dopaminergic drugs for 3 weeks. Dosing and methodology of the test were the same as previous studies, but blood samples were collected every 10 minutes instead of every 15 minutes for 1 hour. The authors found a significantly higher growth hormone response to clonidine in IPD than in the MSA group, but the frequency of patients with positive and negative CGHT did not differ between the two groups. The sensitivity of a negative CGHT for diagnosing MSA was $73 \%$, whereas its specificity was $57 \%$.

Lee et $\mathrm{al}^{17}$ performed CGHT on a larger sample of patients than previous studies and compared the diagnostic accuracy of the CGHT with that of anal sphincter electromyography. Twenty-one IPD, 23 MSA-P and 22 MSA-C patients were enrolled. Antiparkinsonian medication was stopped 48 hours before the CGHT. The clonidine test was performed as previously described. ${ }^{13}$ The authors reported that CGHT had a sensitivity of $78.3 \%$ for the diagnosis of MSA-P and $63.6 \%$ for the diagnosis of MSA-C with a specificity of $85.7 \%$ versus IPD. A reduced growth hormone response to arginine predicted MSA-P with $85.7 \%$ accuracy and MSA-C with $82.4 \%$ accuracy. The sensitivity of CGHT was lower and its specificity higher than anal sphincter electromyography, but combined testing increased specificity and positive predictive value, thereby enhancing diagnostic accuracy in the diagnosis of MSA. Table 1 shows the sensitivity, specificity and positive predictive values derived from different studies on CGHT. 
Despite some discrepancies possibly due to different pharmacological treatment or small numbers of patients in some studies, there is evidence of a reduced growth hormone response to clonidine in MSA versus IPD patients. It is known that adrenergic cells of the ventrolateral medulla project to the hypothalamus and this pathway may influence GHRH release. ${ }^{10}$ A loss of these neurons could explain the lack of growth hormone response to clonidine, an $\alpha_{2}$-adrenoceptor agonist. This is a common finding in MSA, where a loss of catecholaminergic neurons of the intermediate reticular formation of ventrolateral medulla has been clearly demonstrated. 18

\section{Arginine Growth Hormone Test (AGHT) in the Differential Diagnosis of IPD and MSA}

In a recent study, Pellecchia et al ${ }^{19}$ compared growth hormone response to three different stimuli, clonidine, arginine and GHRH+arginine, in 12 MSA, 10 IPD and 30 healthy controls. None of the patients had been taking antiparkinsonian medication for at least 1 month before the study. All subjects had a normal response to the GHRH+arginine test and displayed normal levels of IGF-I and IGF binding protein 3, allowing exclusion of growth hormone deficiency. After clonidine, growth hormone increased in controls and IPD patients but not in MSA patients. CGHT distinguished MSA from IPD with a sensitivity of $80 \%$ and a specificity of $75 \%$. After arginine, growth hormone increased in controls and IPD patients but not in MSA patients. The arginine growth hormone test (AGHT) distinguished MSA from IPD patients with a sensitivity and a specificity of $100 \%$. Growth hormone response to arginine was similar in controls and IPD patients. These results suggested that AGHT was more accurate than CGHT in differentiating MSA from IPD.

It is likely that the impaired growth hormone response to arginine in MSA resulted from abnormalities in the cholinergic pathways involved in growth hormone secretion. The hypothalamic arcuate nucleus and median eminence are richly innervated by the cholinergic neuron system. ${ }^{20,21}$ An intrahypothalamic cholinergic pathway similar to the tuberoinfundibular dopaminergic pathway has been demonstrated in both animals and humans and may be important in some effects of the cholinergic drugs on somatotropic function. ${ }^{10,20,21}$

Evidence of an intrahypothalamic cholinergic defect in MSA arises from clinical and pathologic studies. ${ }^{22,23}$ Biochemical analysis of the hypothalamus from deceased patients with MSA showed reduction of acetylcholine-transferase activity, a marker for cholinergic neurons. ${ }^{22}$ An altered vasopressin response to cholino-mimetic agents has been described in patients with MSA, suggesting an impairment of intrahypothalamic cholinergic neurons. ${ }^{23}$ However, we cannot exclude that the lack of growth hormone response to arginine in MSA patients is due to a loss of extrinsic cholinergic pathways. In fact, ascending projections to hypothalamus arise from brainstem cholinergic nuclei, such as pedunculopontine and laterodorsal tegmental nuclei, that are impaired in MSA. ${ }^{24}$

\section{Conclusions}

Evidence that CGHT may assist in the differential diagnosis of MSA from IPD arises from several studies. AGHT has recently emerged as a promising tool showing higher diagnostic accuracy than CGHT, but these results need to be confirmed by further studies on larger populations.

The abnormal response to clonidine and arginine in MSA but not in IPD suggests specific involvement of noradrenergic and cholinergic pathways involved in the regulation of growth hormone release. Therefore, these tests provide further insight into central neurotransmitter abnormalities in MSA. All the reviewed studies used clinical diagnostic criteria as a gold standard to evaluate if growth hormone response to clonidine and arginine was different in clinically defined MSA and IPD. The lack of a pathological confirmation of the cases studied may be a limitation of these studies.

Growth hormone stimulation tests provide a low cost and practical method for accurately distinguishing MSA from IPD. Future studies are warranted to assess the validity of growth hormone stimulation tests in the differential diagnosis of other forms of parkinsonism.

\section{References}

1. Gudmundsson KR. A clinical survey of parkinsonism in Iceland. Acta Neurol Scand 1967;43:1-61.

2. Kessler II. Epidemiologic studies of Parkinson's disease. 3. A community-based survey. Am J Epidemiol 1972;96:242-254.

3. Marttila RJ, Rinne UK. Epidemiology of Parkinson's disease in Finland. Acta Neurol Scand 1976;53:81-102.

4. Forno LS. The Lewy body in Parkinson's disease. Adv Neurol 1987;45:35-43

5. Hughes AJ, Daniel SE, Kilford L, Lees AJ. Accuracy of clinical diagnosis of idiopathic Parkinson's disease: a clinico-pathological study of 100 cases. J Neurol Neurosurg Psychiatry 1992;55:181-184.

6. Quinn N. Multiple system atrophy. In: Marsden CD, Fahn S, eds. Movement disorders III. London, UK: Butterworth-Heinemann; 1994. 262-281.

7. Papp MI, Kahn JE, Lantos PL. Glial cytoplasmic inclusions in the CNS of patients with multiple system atrophy (striatonigral degeneration, olivopontocerebellar atrophy and Shy-Drager syndrome). J Neurol Sci 1989;94:79-100.

8. Wenning GK, Colosimo C, Geser F, Poewe W. Multiple system atrophy. Lancet Neurol 2004;3:93-103.

9. Colosimo C, Albanese A, Hughes AJ, de Bruin VM, Lees AJ. Some specific clinical features differentiate multiple system atrophy (striatonigral variety) from Parkinson's disease. Arch Neurol 1995;52:294-298.

10. Muller EE, Locatelli V, Cocchi D. Neuroendocrine control of growth hormone secretion. Physiol Rev 1999;79:511-607.

11. Giustina A, Veldhuis JD. Pathophysiology of the neuroregulation of growth hormone secretion in experimental animals and the human. Endocr Rev 1998;19:717-797.

12. Growth Hormone Research Society. Consensus guidelines for the diagnosis and treatment of adults with growth hormone deficiency: summary statement of the Growth Hormone Research Society Workshop on Adult Growth Hormone Deficiency. J Clin Endocrinol Metab 1998;83:379-381.

13. Kimber JR, Watson L, Mathias CJ. Distinction of idiopathic Parkinson's disease from multiple-system atrophy by stimulation of growth-hormone release with clonidine. Lancet 1997;349:1877-1881. 
14. Clarke CE, Ray PS, Speller JM. Failure of the clonidine growth hormone stimulation test to differentiate multiple system atrophy from early or advanced idiopathic Parkinson's disease. Lancet 1999;353:1329-1330.

15. Tranchant C, Guiraud-Chaumeil C, Echaniz-Laguna A, Warter JM. Is clonidine growth hormone stimulation a good test to differentiate multiple system atrophy from idiopathic Parkinson's disease? J Neurol 2000;247:853-856.

16. Strijks E, van’t Hof M, Sweep F, Lenders JW, Oyen WJ, Horstink MW. Stimulation of growth-hormone release with clonidine does not distinguish individual cases of idiopathic Parkinson's disease from those with striatonigral degeneration. J Neurol 2002;249:1206-1210.

17. Lee EA, Kim BJ, Lee WY. Diagnosing multiple system atrophy with greater accuracy: combined analysis of the clonidine-growth hormone test and external anal sphincter electromyography. Mov Disord 2002;17:1242-1247.

18. Benarroch EE, Smithson IL, Low PA, Parisi JE. Depletion of catecholaminergic neurons of the rostral ventrolateral medulla in multiple systems atrophy with autonomic failure. Ann Neurol 1998;43:156-163.

19. Pellecchia MT, Pivonello R, Salvatore E, Faggiano A, Barone P, De Michele G, Lombardi G, Colao A, Filla A. Growth hormone response to arginine test distinguishes multiple system atrophy from Parkinson's disease and idiopathic late-onset cerebellar ataxia. Clin Endocrinol (Oxf) 2005;62:428-433.

20. Tago H, McGeer PL, Bruce G, Hersh LB. Distribution of choline acetyltransferase-containing neurons of the hypothalamus. Brain Res 1987;415:49-62.

21. Caffe AR. Architecture of the mammalian pituitary cholinergic system with observations on a putative blood acetylcholine sensor. Histol Histopathol 1996;11:537-551.

22. Spokes EG, Bannister R, Oppenheimer DR. Multiple system atrophy with autonomic failure: clinical, histological and neurochemical observations on four cases. J Neurol Sci 1979;43:59-82.

23. Norbiato G, Bevilacqua M, Righini V, Chebat E, Vago T, Bertora P, Castelli L, Mangoni A. Altered vasopressin response to metoclopramide in multiple system atrophy: evidence of a cholinergic defect in the hypothalamus. Acta Neurol Scand 1992;85:299-303.

24. Benarroch EE, Schmeichel AM, Parisi JE. Depletion of mesopontine cholinergic and sparing of raphe neurons in multiple system atrophy. Neurology 2002;59:944-946.

\section{Author Affiliations}

Maria Teresa Pellecchia, MD, Department of Neurological Sciences, University of Naples "Federico II", Naples, Italy

Rosario Pivonello, MD, Department of Molecular and Clinical Endocrinology and Oncology, University of Naples "Federico II', Naples, Italy

Annamaria Colao, MD, Department of Molecular and Clinical Endocrinology and Oncology, University of Naples "Federico II", Naples, Italy

Paolo Barone, MD, Department of Neurological Sciences, University of Naples “Federico II”, Naples, Italy 